\title{
A Customizable Multilingual Chatbot System for Customer Support
}

\author{
Vinothini Kasinathan', Aida Mustapha ${ }^{2}$ and Chow Khai Bin ${ }^{1}$ \\ ${ }^{1}$ Asia Pacific University of Technology and Innovation, Malaysia \\ vinothini@apu.edu.my; tp040968@mail.apu.edu.my \\ ${ }^{2}$ Universiti Tun Hussein Onn Malaysia, Malaysia \\ aidam@uthm.edu.my \\ *Correspondence: vinothini@apu.edu.my
}

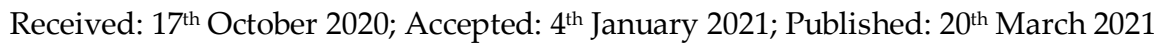

Abstract: Implementing third-party services to develop a chatbot is not cost-effective for many small and medium enterprises especially considering that many services only supports one language at a time. To address this gap, this paper proposes a multilingual chatbot system that will allow companies and organizations to customize and deploy their own multilingual chatbot service with two extended features, which are live chat and ticketing system. The chatbot will also be able to understand and reply in English, Malay, and Chinese as well as customizable given the dialogue shell and knowledge base. To achieve this, the development uses TypeScript for frontend web application while Go as the backend development. The development language for mobile application is Dart and the User Interface (UI) library is React. Finally, the database management system used is MongoDB. The developed prototype is then evaluated via a survey questionnaire and the findings suggested that the proposed system would be able to assist small and medium-sized business and organizations to deploy their own chatbot system as an alternative to existing customer services.
\end{abstract}

Keywords: Conversational agents; Chatbots; Multilingual; Customer services

\section{Introduction}

Chatbots are computer programs that interact with users using natural languages. This technology started in the 1960's with the aim was to see if chatbot systems could fool users that they were real humans [1]. However, chatbot systems are not only built to mimic human conversation, it is also useful in several fields such as education, information retrieval, business, and e-commerce. Chatbot applications streamline interactions between people and services, enhancing customer experience [2]. Business Insider experts predict that by 2020, 80\% of enterprises will use chatbots [3]. According to Spiceworks study, the highest percentage of department using chatbot in the daily operation is the IT department followed by Administrative department and Customer Service department [4]. Figure 1 shows the departments utilizing chatbots within organization.

The use of chatbots also bring more savings and efficiency to an organization. The right method can significantly lower the customer service costs in terms of manpower and infrastructure, thus making it possible to reach higher profitability. Chatbots are able to maintain the same service quality, such as lowering the possibility of a mistake by a salesperson or any other department. Chatbots would make marketing and advertising activities a lot easier since the bot now engage in one-to-one dialogue with the consumer and even providing suggestions [5]. Access to greater detail of products and a greater detail of the consumer preferences are also important for the chatbots to increase the likelihood of a sale, hence generating a greater revenue than the traditional methods. Figure 2 shows the potential annual US salary savings created by chatbots. In some industries, it 
reaches up to $60 \%$ saving to implement a chatbot [3]. More emerging industries are taking part in using chatbots such as in journalism [6], pharmaceutical [7], tourism [8].

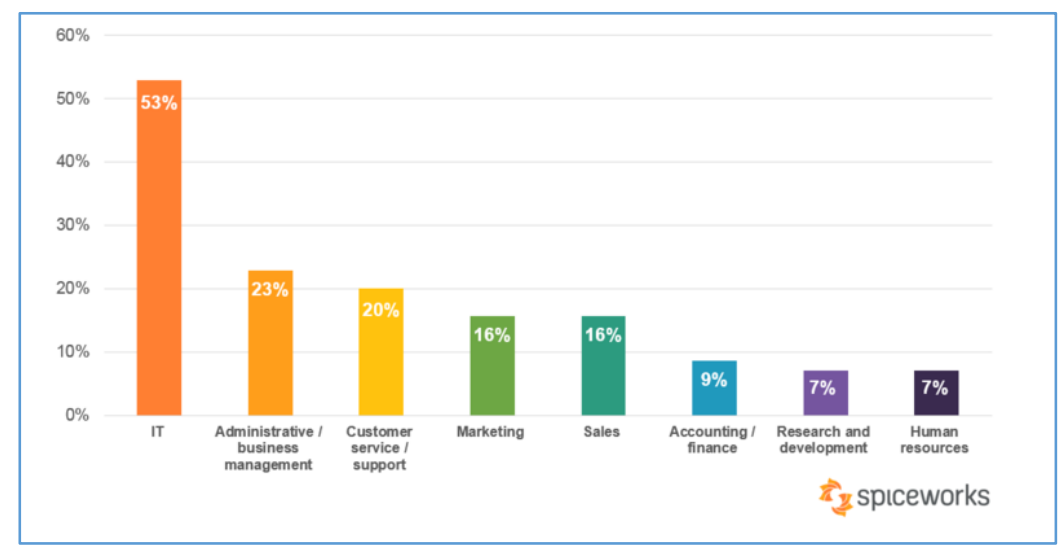

Figure 1. Departments utilizing chatbots within organization [4]

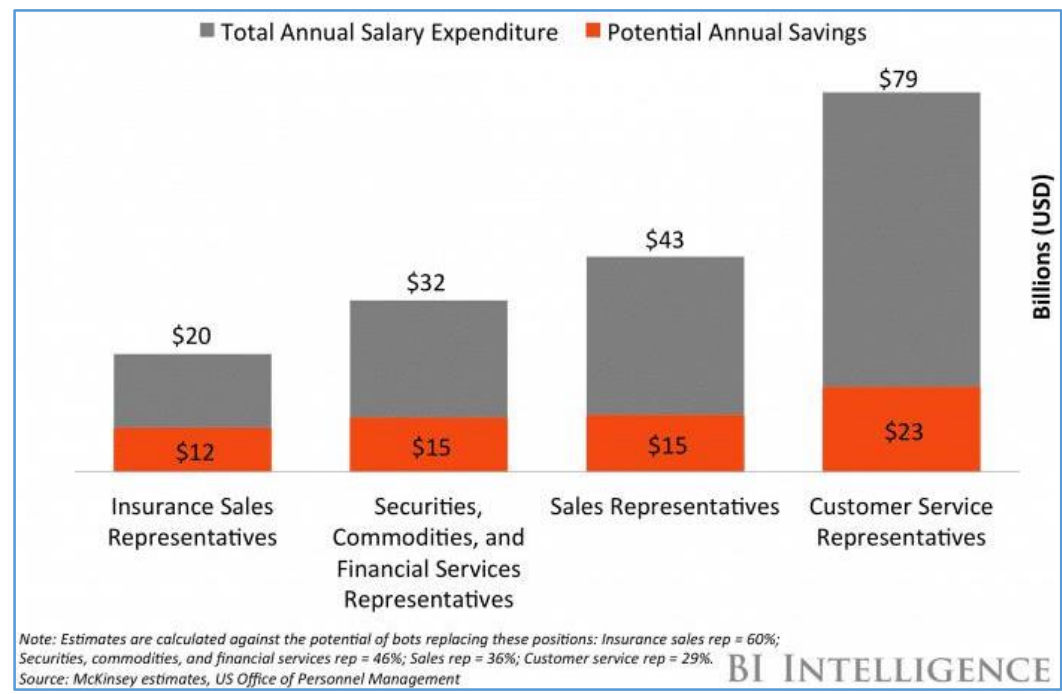

Figure 2. Potential annual US salary savings created by chatbots [3]

Multilingual conversational bot or chatbot is important especially in Malaysia because Malaysia is a multiracial country that is well-known for rich dialects and slang across different social communities. However, a typical chatbot service such as Dialogflow (https://dialogflow.com/) and RasaNLU (https://rasa.com/) only supports one language at a time and the training process is not user-friendly because it requires different dialects for one intents. It is also difficult for these highlevel chatbot services to implement extended services such as live chat and ticketing function. Implementing third-party services will be even more complicated because it is often non-cost effective for small and medium enterprise due to high development cost.

This paper proposed a multilingual chatbot that allow customers to chat in mixed language as like they are chatting with a real human. The chatbot will have extended features such as live chat and ticketing system will improve the chatbot system by allowing customer's route to real support agents when they require further assistance or complains. With email piping, the customers will receive email when the agent respond to the ticket and the customer can reply to the email directly. The chatbot flow and behaviour can be customized with a flow chart and the chatbot flow is expressive with flow chart view. With injection script, clients can implement the chatbot service easily and it does not require any prerequisite before implementing.

This paper proceeds as follows. Section 2 presents the materials and methods in developing the prototype, Section 3 presents the evaluation results and finally, Section 4 concludes the paper.

\section{Materials and Methods}

This paper proposed a multilingual chatbot system that will allow companies and organizations to customize and deploy their own multilingual chatbot service with two extended features, which 
are live chat and ticketing system. The live chat system, ticketing system and tracker system are optional and extended features of the chatbot. Live chat system allows agent to chat with their customer in real-time if the chatbot does not to understand the customer needs. The ticketing system is useful when there are no online agents or and it is aimed to use for solving customers' problems that cannot be solved instantly. Once a ticket is generated, the agents will be able to reply to the ticket via the admin panel and the customers will be able to track and response to the ticket via email.

The system allows the organization admin account to customize the chatbot flow and behaviour through the admin panel. The chatbot can be customized and represented in a simple flow chart and all changes will reflect to their deployed web application. The admin account also can view reports and manage agents. Agents are the human support agent of the system. Agents can be managed by admin and they have access to the agent panel. The agents are allowed to view customer queue and reply to their customers in real-time though live chat services. The agents are also allowed to generate a ticket and reply to a ticket to in order to track a case that cannot be resolved instantly. The agents can view the list of customer interaction or action data to process a new order or feedbacks.

The chatbot will not only understand and reply in English, it can also capable of replying in Malay and Chinese. The client can implement the chatbot service into their current existing web application without hassle. The chatbot can be implemented in a normal HTML site as well as CMS sites such as Wordpress, Wix, Weebly, and others. The client does not require technical knowledge to implement the chatbot service as the system is provided with a step-by-step guide for different type of web application.

\subsection{System Design}

The proposed multilingual chatbot system is designed with multiple user roles, which includes an admin, an agent, and a customer. Figure 3 shows the use case diagram for the proposed chatbot system.

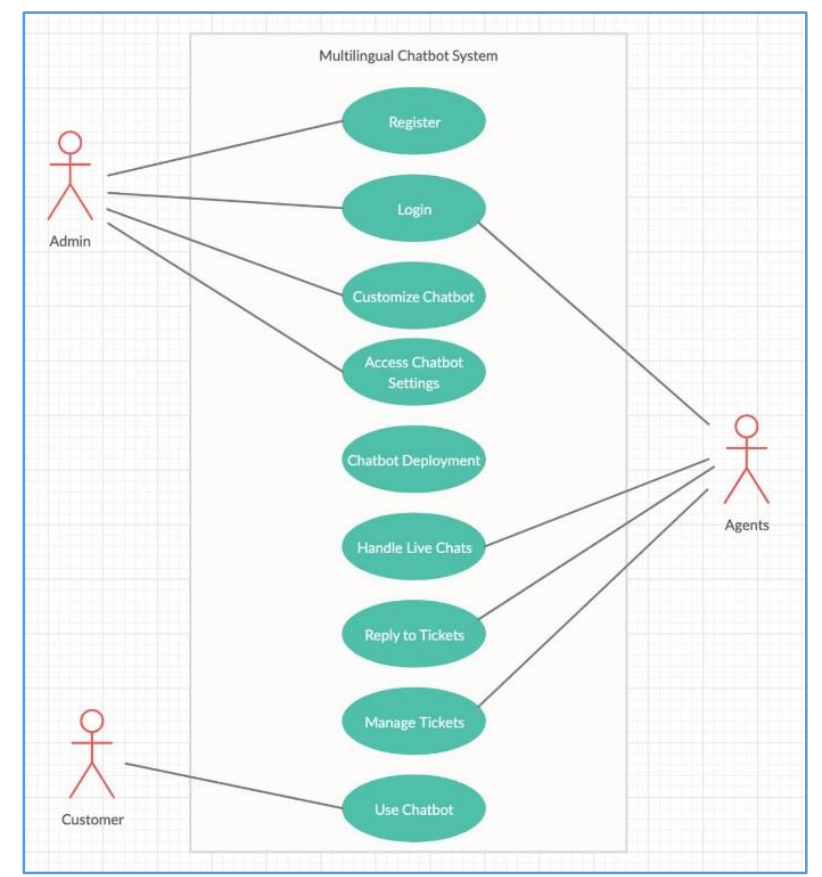

Figure 3. Use case diagram of the multilingual chatbot system

The admin will be able to setup or create a new account for their organization uses. To get started, they are required to verify the email domain of the organization. After verification success, they will be redirected to the chatbot admin panel which consists of all kind of chatbot configuration and behaviour customization. On the chatbot customization panel, admin can customization the chatbot behaviour based on a flow chart. A flow chart is a good choice for displaying and customizing a flow-based chatbot activity. 
During the customization process, admin can optionally provide the context for different languages in order to have multilingual chatbot support. Currently the support languages are English, Malay, and Chinese, whereby this combination is well suitable for Malaysian and Singaporean companies and can be expanded to support more languages as it grows. After customizing the chatbot, the admin can optionally configure the chatbot avatar and appearance on the chatbot menu. After completion, the system will generate a Javascript injection code that will be used for web app deployment. The deployment is as easy as copying one line of html script block into current html file.

After injecting the code, end users or customers should be able to view the chatbot system built on top of the web application. The end users can freely interact with the chatbot and play with it. The chatbot system also supports multiple enterprise use-case like live chat system and ticketing system. When there are live agents available, the customers can chat to live agents during working hour or optionally create a report ticket if no agents are available. In general, the customers are able to ask questions, choose language, make choices, and view results or answer.

\subsection{Development Requirement}

Go is chosen as the language of backend development. Go is syntactically similar to C, but with memory safety, garbage collection, structural typing, and CSP-style concurrency. Go has the best performance when compared with other famous backend languages such as PHP, Java and NodeJS.

As for the frontend development requirements, TypeScript with TypeScript Syntax Extension is chosen as the development language for frontend web application. TypeScript is an open-source programming language developed and maintained by Microsoft. It is a strict syntactical superset of JavaScript, and adds optional static typing to the language. JavaScript is chosen as the development language for client-side web application script injection. JavaScript is one of the core technologies of the World Wide Web. JavaScript enables interactive web pages and is an essential part of web applications. Dart is chosen as the development language for mobile application. Dart is a clientoptimized programming language for cross-platform applications.

For library and tools, React is chosen as the library of frontend web application for building user interfaces. React is used as a base in the development of single-page or mobile applications. Flutter is chosen as the tool of cross-platform mobile application. Flutter is an open-source UI software development kit created by Google. Next, Docker is chosen to make it easier to create, deploy, and run applications by using containers. Containers allow a developer to package up an application with all of the parts it needs, such as libraries and other dependencies, and ship it all out as one package.

Finally, the database management system used is MongoDB, which is chosen as the database management system since it is a cross-platform document-oriented database program. Classified as a NoSQL database program, MongoDB uses JSON-like documents with schema, hence we can build applications faster, handle highly diverse data types, and manage applications more efficiently at scale.

\subsection{Prototype Development}

The target users of the system are organization or enterprise with web application. Figure 4 shows the feature for customization of page. This system allows users, who is the organization administrator, to customize the avatar for their company and even change the colours. Admin can customize the chatbot using only drag and drop interface. The main benefit to an organization is that it will save cost by reducing the amount of work of the customer support agents. The chatbot developed can be extended to have more features, and interactive chatbot in a web application leads to more customer engagement. The customers have a good experience when asking questions through chatbot. 


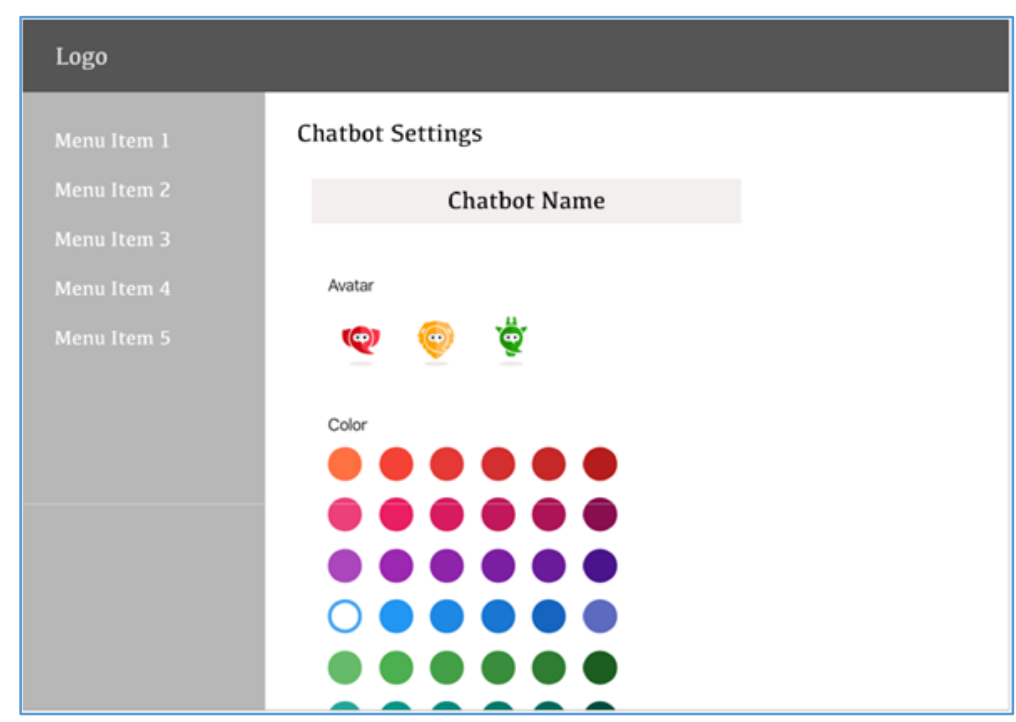

Figure 4. Chatbot customization page

Figure 5 shows the chatbot deployment page. Organizations can sign up in this page in order to use the chatbot service. Admin can access the deployment guide for multiple web applications. Figure 6 shows the Sample deployment of keywords to answer users' questions.

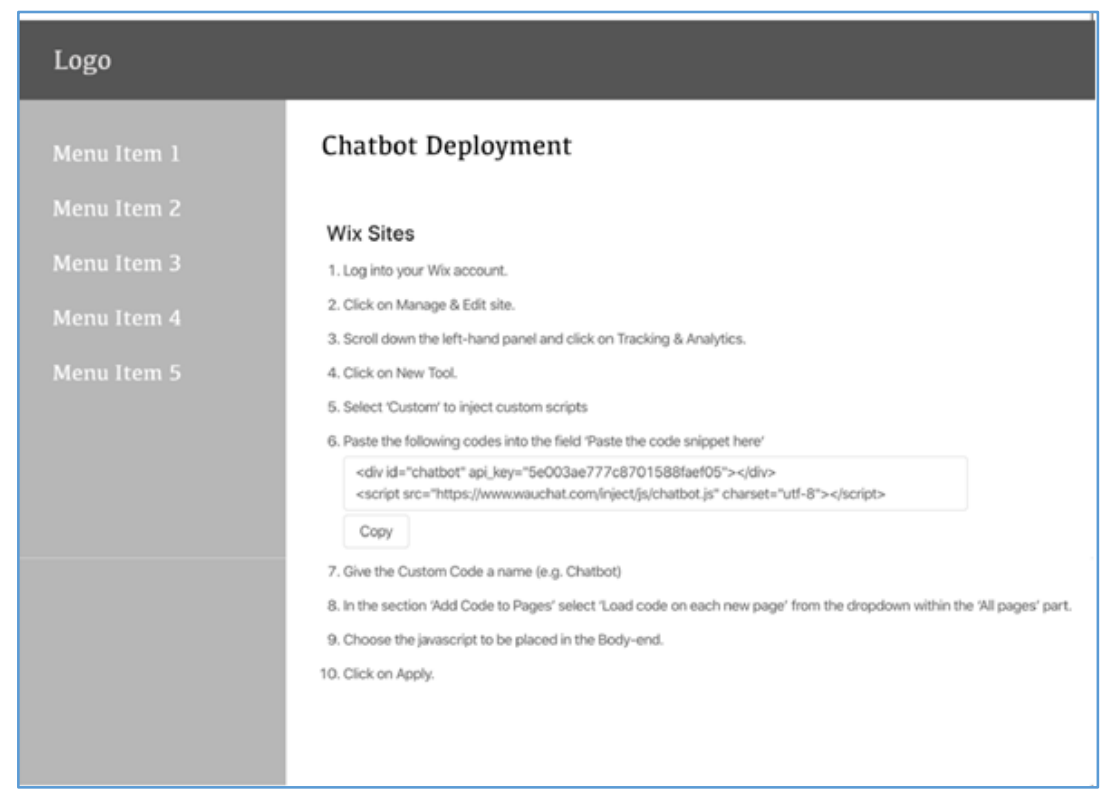

Figure 5. Chatbot deployment page

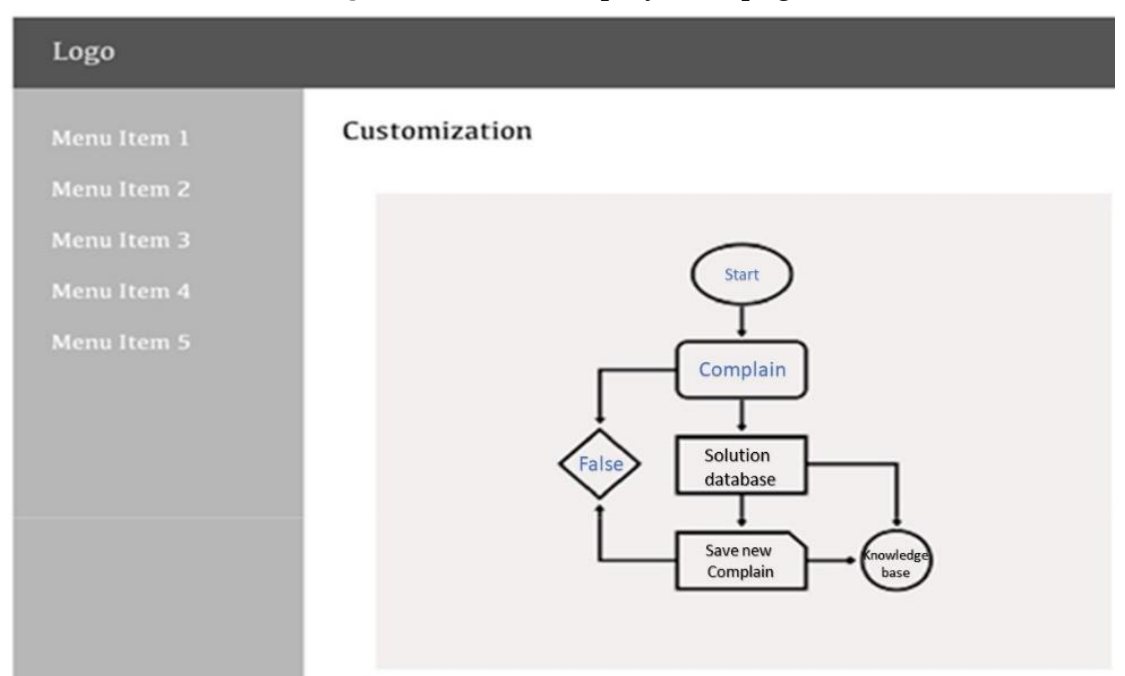

Figure 6. Sample deployment of keywords to answer users' questions 


\section{Evaluation}

Based on [9], the multilingual chatbot system is evaluated based on survey questionnaire. Most of the data is based on measured values and can be checked by others because numerical data is less open to ambiguities of interpretation. It is also cheaper when compared with other tools and sending the Google form to respondents. There was a total of 27 respondents who participated in the survey from a total of 50 survey invitations sent out to IT departments in various companies within Selangor, Malaysia from the state database ${ }^{1}$.

The first question was the background of the users web application used in the organization. The results are shown in Figure 7. Majority of the respondents are using static or dynamic web application. The chatbot service injection can be focused on static or dynamic web application followed by Wordpress and Wix. There are a very small amount of respondents does not have a web application which left them impossible to implement the chatbot service.

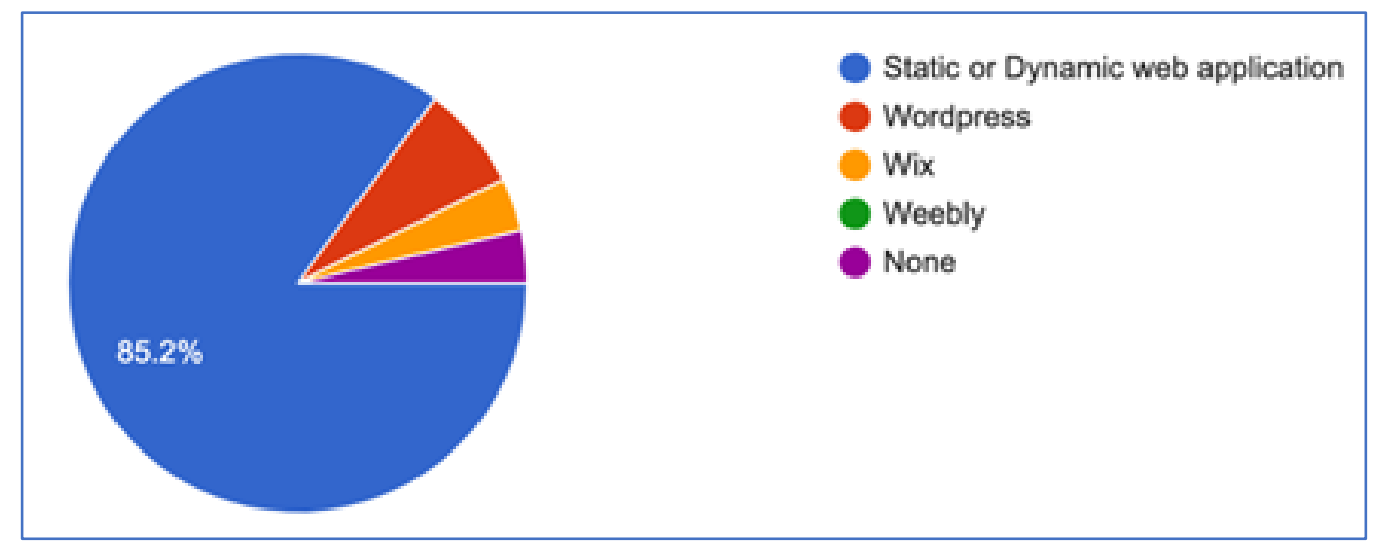

Figure 7. Users preference towards web application

The next question was which customer services are organization providing and the results, as anticipated, showed that chatbot is not a popular choice. The idea of adding in chatbot on website will be good way. Most respondents are provider phone service followed by email and walk-in service. There are about $33.3 \%$ of them receiving online form. Only about $12.5 \%$ of the respondents are providing live chat services and there are none of them implemented chatbot service. All respondents can adapt to the new chatbot service and the live chat options can be replaced for some of them who're providing live chat services. The summary is shown in Figure 8.

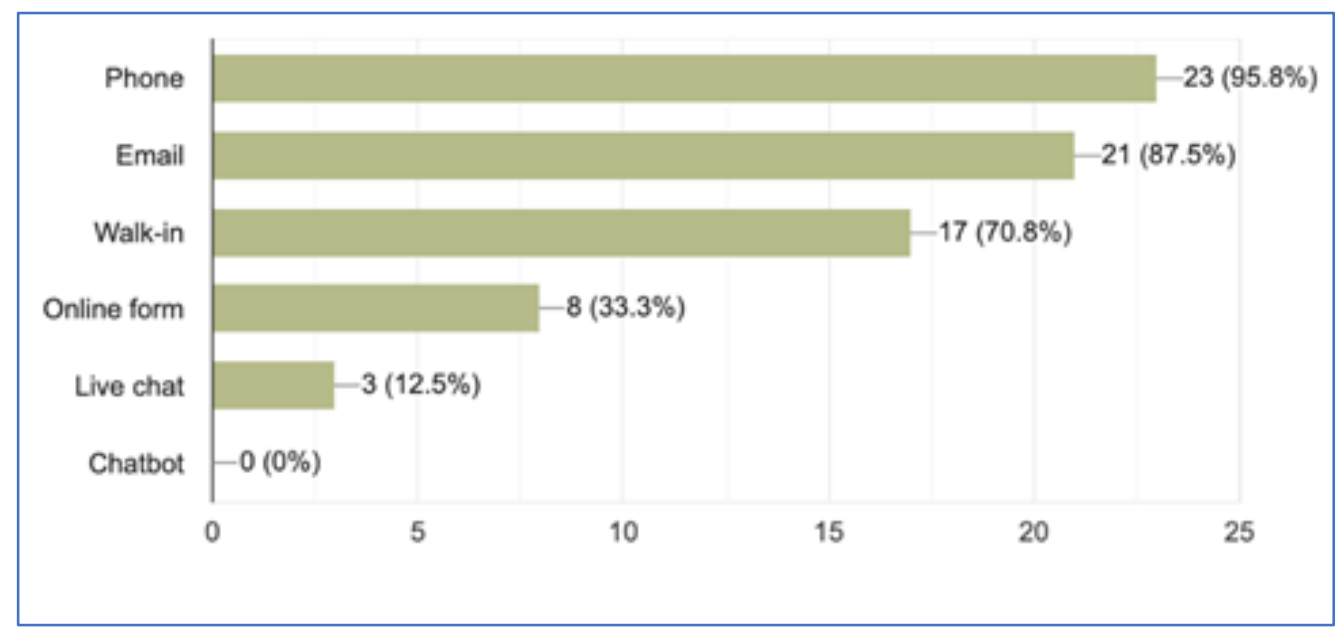

Figure 8. Common customer services provided on website

Next, Figure 9 shows that majority of the respondents are struggling with customer service response times because they require human support agents to reply to the customers. The customers may have to queue for a long time during peak hour, and the support agents are only available during

\footnotetext{
${ }^{1}$ https://www.businesslist.my/state/selangor
} 
working hours. In this case, they can implement a chatbot service which will automatically reply to their customers instantly $24 / 7$.

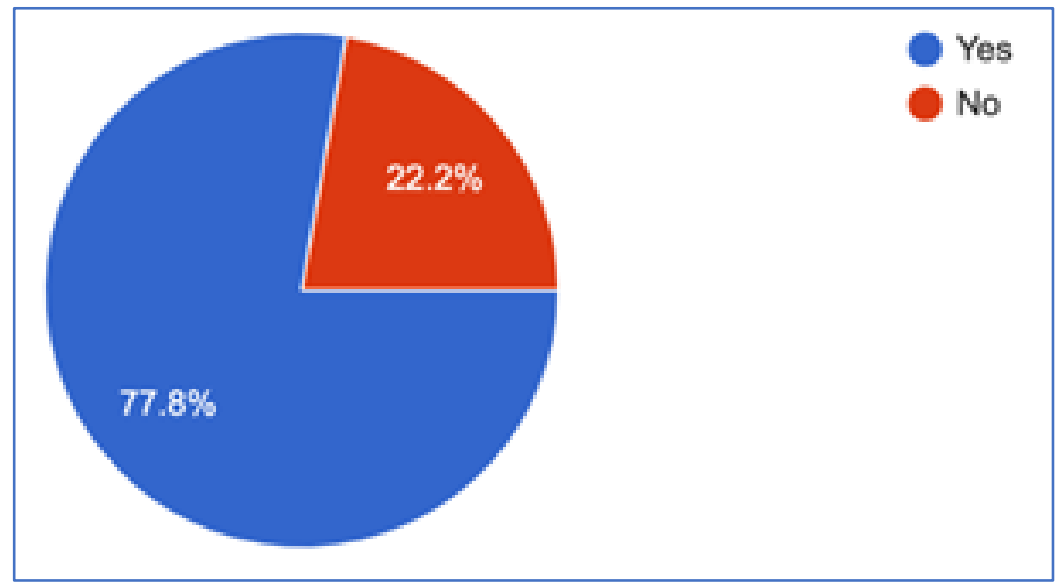

Figure 9. Shows organization are happy using chatbot as customer service

According to Figure 10, majority of the respondents are interested to capture customer service interaction data. Capturing customer interaction data is difficult to achieve data consistency and require longer time to analyze if doing manually. The system can be improved by capturing customer service interactions by tracking all the customer interaction including chatbot and live chat conversation.

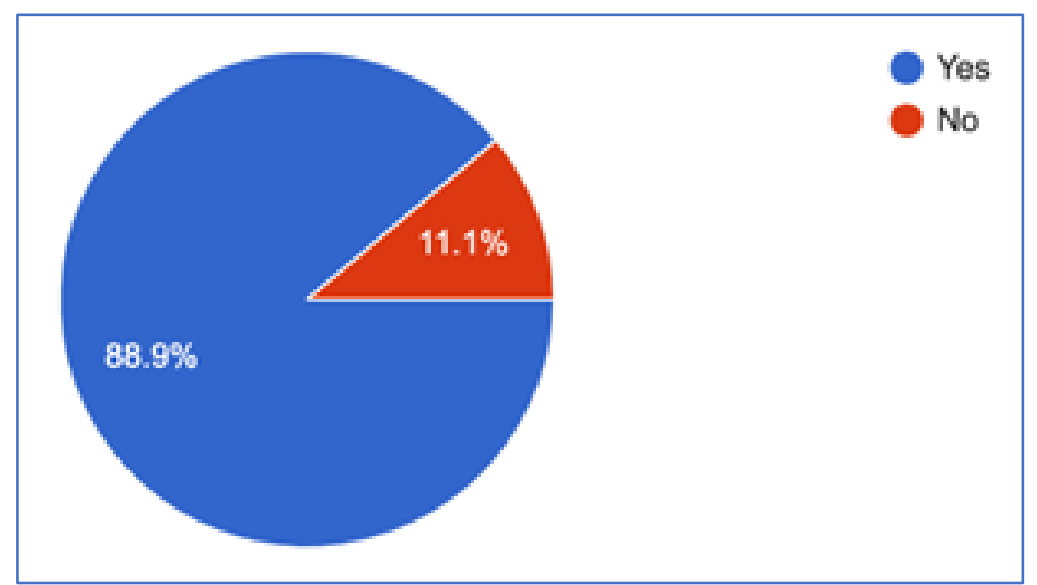

Figure 10. Users like using chatbot

As shown in Figure 11, most of the respondents are interested in implementing a chatbot services in their web application. Which means there's a great chance that they will try out or implement the chatbot service and this makes the project feasible to continue working on.

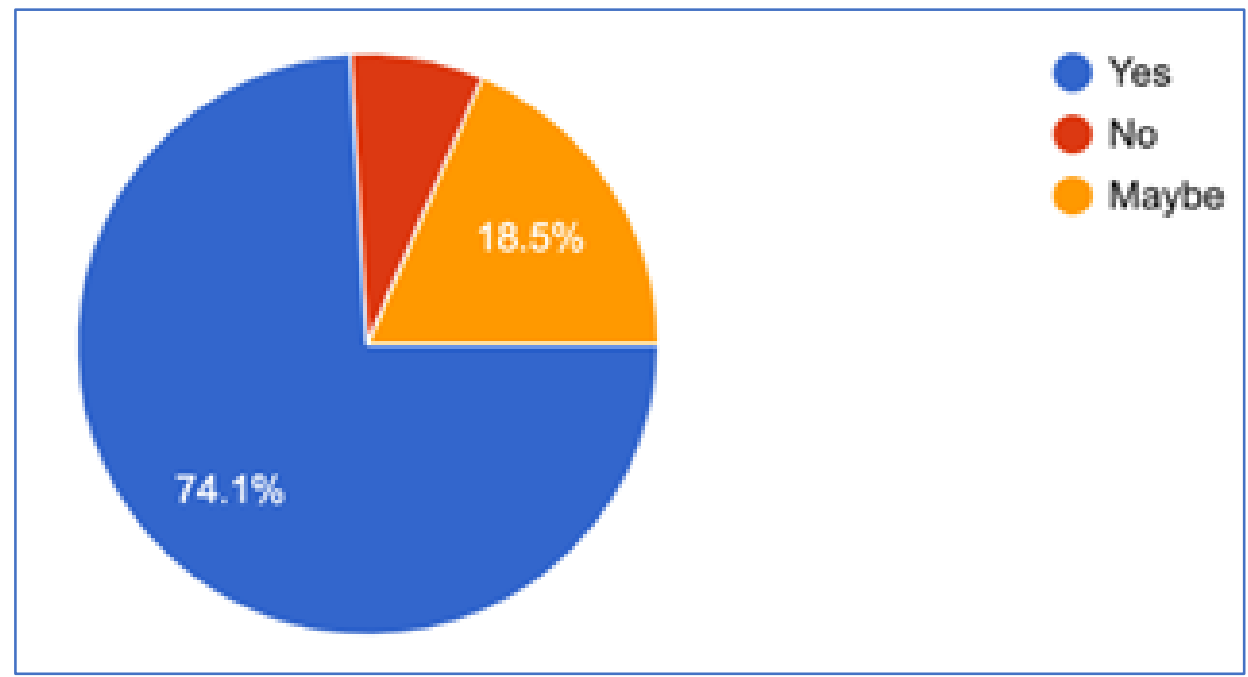

Figure 11. Possible implementation of chatbots in organization 
Most of the respondents think that responding to their customer instantly at any time is important for a chatbot service and they expect a chatbot can answer some fact-based questions. There are $72 \%$ of them thinks that customization and integration of chatbot is equally important as multilingual support while $64 \%$ of respondents thinks that having a live chat support integrated into the chatbot system is important. This data will helps to study about which category or features to focus the most and the least. The findings are shown in Figure 12.

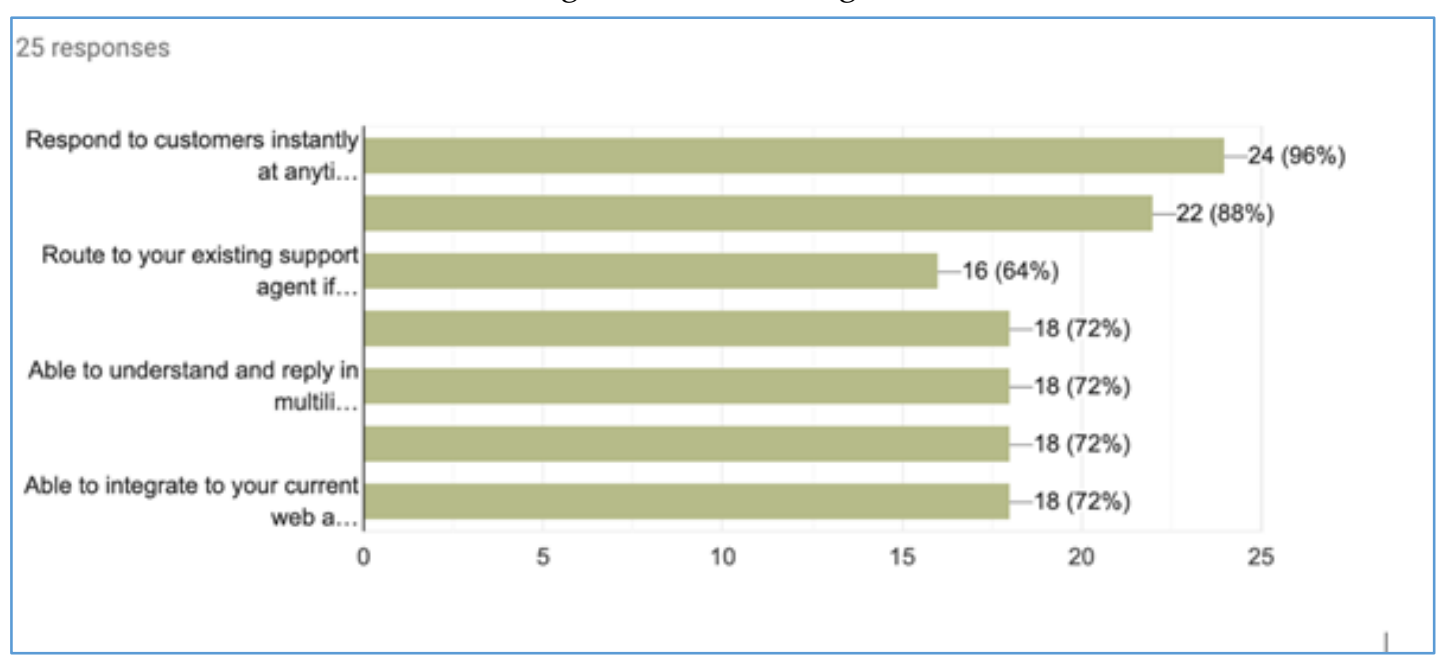

Figure 12. Chatbot features liked by the users

Finally, most respondents think that having a mobile application for customer support agent is useful for them to reply to customer instantly on-the-go as shown by Figure 13. In the chatbot system, a mobile application will be developed for agents to allow them reply to live chat and tickets.

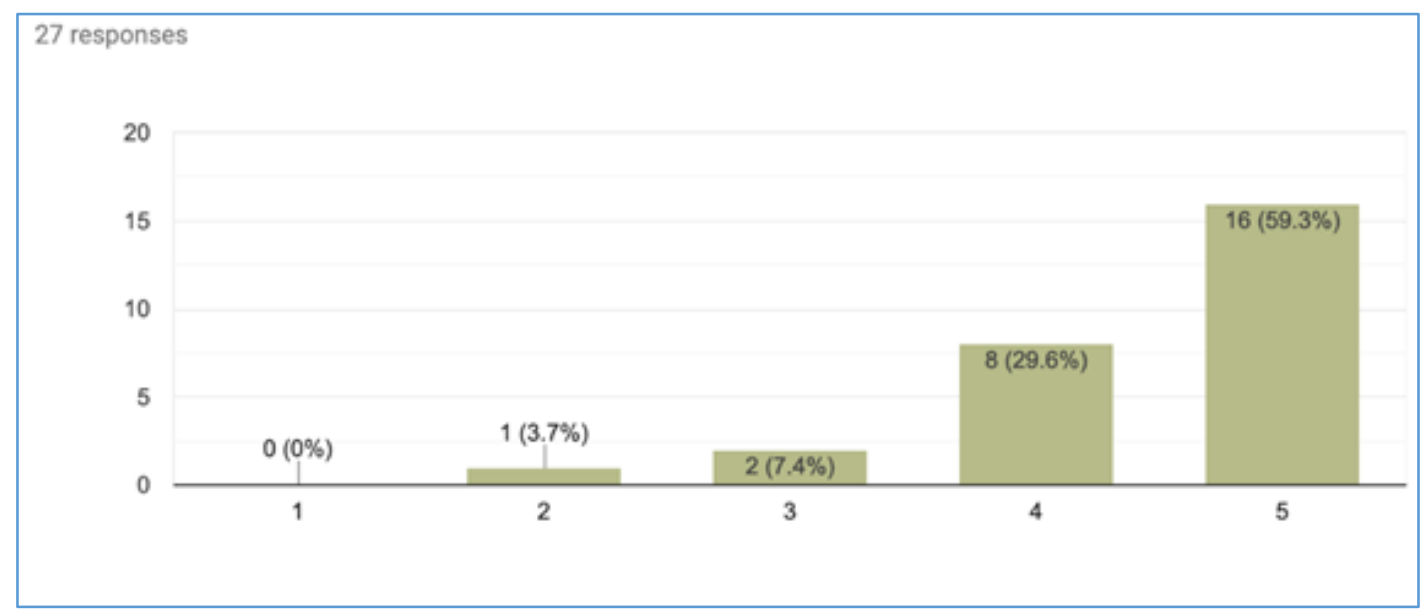

Figure 13. Mobile application is useful for chatbot to reply in real time

\section{Conclusion}

This paper proposed a customizable, multilingual chatbot system to assist organization to selfdevelop their own chatbot as customer service. A live chat and ticketing system is integrated into the chatbot service to allow agents to provide further assistance to their customers. Customers can choose to queue for a live chat service and the agents can join the live chat on their respective agent panel or mobile application. For problems that cannot be solved instantly, ticketing system will come into play and agent can generate a tracking ID for the particular customer with problem description. The agents can reply to the tickets through their panel and customers will be notified through their email address.

Although the system has been developed, there are some limitations should be considered to improve in the future. But achieving multilingual chatbot is a big step towards the future and having it easy to deploy is important too. The checklist of limitation/improvements are:

- Add support for more languages and not only English, Malay, and Chinese 
- Add support for speech to text and text to speech

- Improving languages models to achieve better matching accuracy

- Implement deep learning algorithm instead of matching keywords

It is hoped that this system would be able to assist small and medium-sized business and organizations to deploy their own chatbot system as an alternative to existing customer services.

\section{Acknowledgment}

This research is supported by Asia Pacific University of Innovation and Technology.

\section{References}

[1] Shawar B.A. and Atwell E. (2007). Chatbots: are they really useful?. In LDV Forum, Vol. 22, No. 1, pp. 2949.

[2] Almurshidi S.H. and Abu-Naser S.S. (2018). Expert System For Diagnosing Breast Cancer. Al-Azhar University, Gaza, Palestine.

[3] Business Insider Intelligence. (2016). $80 \%$ of businesses want chatbots by 2020. Available: https://www.businessinsider.com/80-of-businesses-want-chatbots-by-2020-2016-12.

[4] Tsai P. (2018). Data snapshot: AI Chatbots and Intelligent Assistants in the Workplace. Spiceworks Blog. Available: $\quad$ https://community.spiceworks.com/blog/2964-data-snapshot-ai-chatbots-and-intelligentassistants-in-the-workplace.

[5] Broeck E.v.d., Zarouali B. and Poels K. (2019). Chatbot advertising effectiveness: When does the message get through?. Computers in Human Behavior, Vol. 98, pp. 150-157.

[6] Veglis A. and Maniou T.A. (2019). Chatbots on the rise: A new narrative in Journalism. Studies in Media and Communication, vol. 7, no. 1, pp. 1-6.

[7] Ahmad N.S., Sanusi M.H., Wahab M.H.A, Mustapha A., Sayadi Z.A. and Saringat M.Z. (2018). Conversational Bot for Pharmacy: A Natural Language Approach, In Proceedings of the 2018 IEEE Conference on Open Systems (ICOS), Langkawi Island, Malaysia, 2018, pp. 76-79, doi: 10.1109/ICOS.2018.8632700.

[8] Ukpabi D.C., Aslam B. and Karjaluoto, H. (2019). Chatbot adoption in tourism services: A conceptual exploration. Robots, Artificial Intelligence, and Service Automation in Travel, Tourism and Hospitality, Emerald Publishing Limited, pp. 105-121.

[9] Maroengsit W., Piyakulpinyo T., Phonyiam K., Pongnumkul S., Chaovalit P. and Theeramunkong T. (2019). A Survey on Evaluation Methods for Chatbots. In Proceedings of the 2019 7th International Conference on Information and Education Technology, pp. 111-119.

(C) 2020 by the author(s). Published by Annals of Emerging Technologies in Computing (AETiC), under the terms and conditions of the Creative Commons Attribution (CC BY) license which can be accessed at http://creativecommons.org/licenses/by/4.0. 